\title{
Flow-field of the Excited Jet and Mixing in the Supersonic Flow
}

\author{
Shenglong $\mathrm{Gu}^{1}$ Lihong Chen ${ }^{2}$ Hongbin $\mathrm{Gu}^{3} \mathrm{Fei}^{4}{ }^{4}$ Zhe Wei ${ }^{5}$ Xinyu Chang ${ }^{6}$ \\ Key Lab of High Temperature Gas Dynamics, \\ Institute of Mechanics, Chinese Academy of Sciences, Beijing 100190, P.R. China
}

\begin{abstract}
The planar laser scattering and gas sampling were used to investigate the mixing of the excited $\mathrm{CO}_{2}$ jet into the supersonic flow in this article. The jet was from the HartmannSprenger tube, which was put into the traditional jet device. The jet was excited at three different frequencies and compared with the base case without excitation. The results shown that the better mixing with less total pressure loss could be obtained in the excited cases. The penetration was the deepest for $900 \mathrm{~Hz}$-excited jet while the number of the large-scale structures was the most in the $\mathbf{5} \mathbf{~ k H z}$-excited case. The excited jet didn't only take effects on the jet shear layer, but also influenced the boundary layer.
\end{abstract}

\section{Nomenclature}

$=$ Frequency

$=$ Dimensionless Frequency

$=$ Sound speed

$=$ Jet diameter

$=$ Tube diameter

$=$ Exit diameter

$=$ Tube Length

$=$ Tube standoff

$=$ Jet Pressure ratio $\left(\mathrm{P}_{\mathrm{j} t} / \mathrm{Pa}\right)$

\section{Introduction}

$\mathrm{T}$

HE efficient mixing and combustion must achieve in the limited space and time for the scramjet engine. The performance of scramjet would be controlled by the process and extent of mixing between fuel and air. It is very difficult to make the fuel penetrate into the supersonic flow deeply for forming the efficient mixing with air, which is the base of the efficient combustion.

Fuel injection could be transverse or parallel je into the main flow. The transverse jet could bring the better penetration with the larger total pressure loss while the parallel jet could cause less total pressure loss with the worse penetration. According to the jet's drawbacks, researchers all over the world developed lots of methods to enhance mixing, such as the excited jet. The excited jet could be the source of disturbance to stimulate the fluctuation in the boundary layer to increase the instability. On the other hand, the excited jet also could amplify the vortex of the jet shear layer to induce large-scale structures.

Randolph $\left[{ }^{1]}\right.$ investigated the effect of a forcing jet transverse into a supersonic cross flow. The frequency of the forcing jet was chosen to be $1 \mathrm{~Hz}$. The results showed $12 \%$ increase in penetration when compared with a steady jet at the same peak exit pressure. Gutmark $\left[{ }^{2]}\right.$ used the Mie scattering technique to visualize the flow field of the excited

\footnotetext{
${ }^{1}$ Ph.D Student,

2 Professor,

3 Associate Professor,

${ }^{4}$ Assistant Professor,

5 Graduate Student,

${ }^{6}$ Professor,
}

Key lab of high temperature gas dynamics, Key lab of high temperature gas dynamics, Key lab of high temperature gas dynamics, Key lab of high temperature gas dynamics, Key lab of high temperature gas dynamics, Key lab of high temperature gas dynamics, gushenglong@imech.ac.cn

lhchen@imech.acn

guhb@imech.ac.cn

lifei@imech.ac.cn

weizhejiji@yahoo.com.cn

xychang@imech.acn

American Institute of Aeronautics and Astronautics 
jet under high frequencies in the Mach 2 supersonic cross flow, and their results showed that the mixing was benefitted with excited jet..

\section{Principle of H-S Tube}

There are many methods to excite jet, and H-S tube is the useful one.

Hartmann developed an acoustic device that generates intense high frequency signal. The device, which was called as the Hartmann-Sprenger tube ( $\mathrm{H}-\mathrm{S}$ tube) later, consists of a supersonic under-expanded jet and a co-axial resonance tube which is closed at another end. The $\mathrm{H}-\mathrm{S}$ tube could produce large amplitude rapid oscillations when the resonance tube was placed in the compressed region of the shock-rhombus. There are several important parameters, which are the pressure ratio of the jet $\mathrm{R}$, the standoff distance $\mathrm{X}$, the tube length $\mathrm{L}$ and the diameter of the jet $D_{j}$ and the tube $D_{t}$, as shown in Figure 1 .

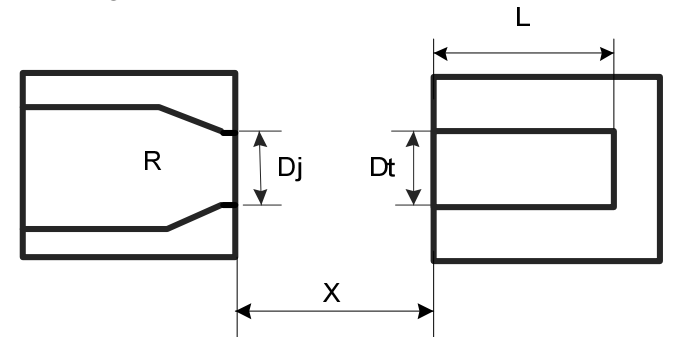

Figure 1 Sketch of the Hartmann-Sprenger tube

Sarobia $^{[3]}$ demonstrated that there were three different modes of the H-S tube depending on the choices of these parameters as mentioned above. The first is the jet instability mode, which was excited for subsonic jets over a large range of $\mathrm{X}$, excited at shear-layer initial instability frequency combined with the fundamental quarter wave of the resonance tube. The second mode, which was called as the jet regurgitant(JRG) mode, occurred due to the periodic swallowing and discharging of the jet flow by the resonance tube at the fundamental tube resonance frequency $(\mathrm{f}=$ $\mathrm{C} / 4 \mathrm{~L}$, where $\mathrm{C}$ is the sound speed in the tube). The third was called as screech mode, which was a high-frequency mode and occurred due to the formation of a normal shock in the front of the resonance tube. The strength and location of this mode was governed by the pressure ratio $\mathrm{R}$ and the standoff distance $\mathrm{X}$.

Compared with the other two modes, the JRG mode is more controllable and can produce stronger oscillation. But the estimated equation of oscillation frequency in the reference had a large error compared with the measured value, especially in the case of the small diameter of tube. Therefore, Gu[4] modified the estimated equation according to the experimental data, in order to design more accurate frequency for the case of small diameter of the resonance tube, as shown in Figure 2 .

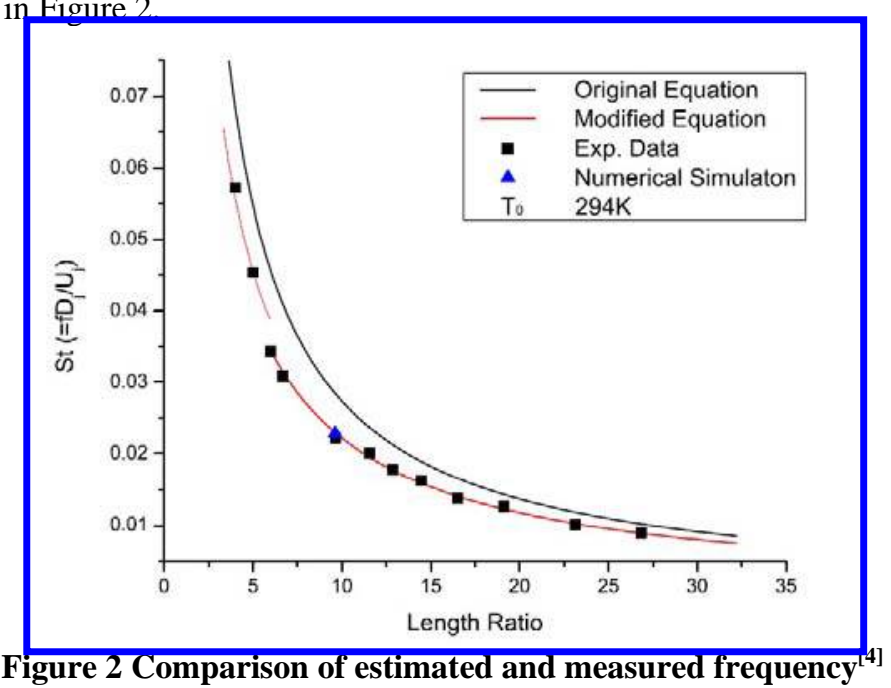




\section{Experimental Setup}

The experiments were performed in the direct-connection supersonic combustion test facility, shown in Figure 3, in the Institute of Mechanics, Chinese Academy of Science. The air flow with high pressure and high temperature which the setup needed was supplied by the heater, and was accelerated to Mach 2.5 by the supersonic nozzle, then entered into the isolator, $1^{\text {st }}$ test section, $2^{\text {nd }}$ test section and nozzle in sequence.

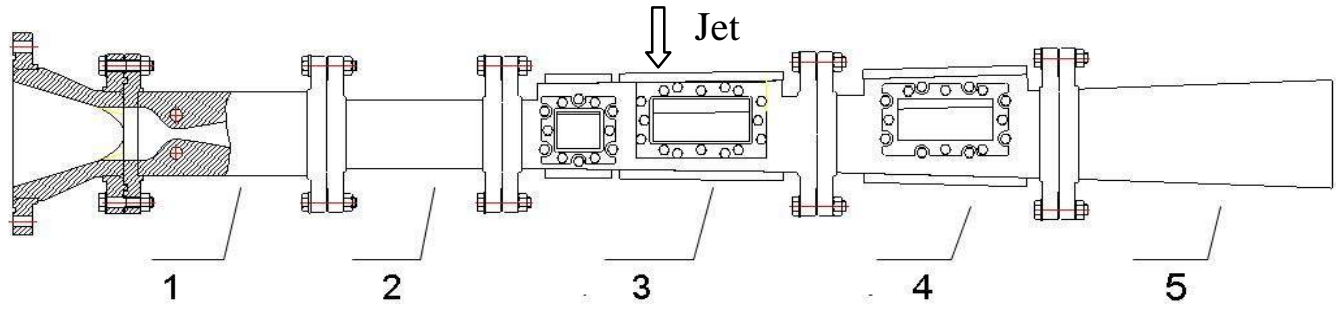

1-Facility Nozzle 2- isolator 3-1 ${ }^{\text {st }}$ test section $4-2^{\text {nd }}$ test section 5-Nozzle Figure 3 Experimental Setup

The structure of the excited jet is shown in the Figure 4, whose exit is about $5 \mathrm{~mm}$. The input to the device is $\mathrm{CO}_{2}$ flow with constant mass flow rate at a certain pressure and temperature. The output is a circular jet with fluctuating flow in the axial direction superimposed on the mean field. The H-S tube was tuned to the JRG mode while the desirable frequencies could be obtained by changing the tube length $\mathrm{L}$ with other parameters fixed. The cases were investigated under excited frequencies at $900 \mathrm{~Hz} 1.3 \mathrm{kHz}$ and $5 \mathrm{kHz}$, which compared with the base case without excitation.
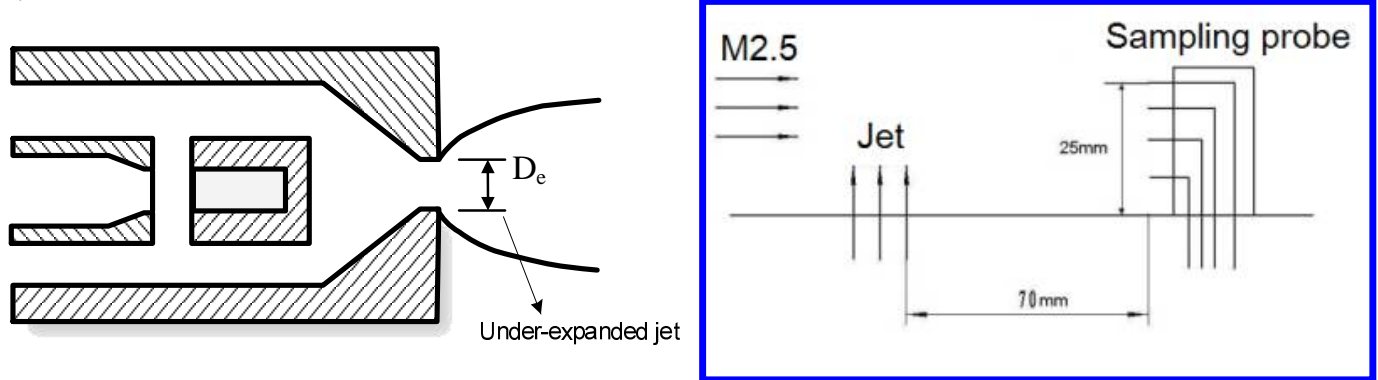

Figure 4 Sketch of the excited jet

Figure 5 Location of the sampling probe

The parameters of main flow in the experiments were Mach 2.5, the total temperature with 960K 980K and the total pressure with $1.26 \mathrm{MPa} \pm 6 \%$. The total pressure and total temperature of $\mathrm{CO}_{2}$ jet were $0.65 \mathrm{MPa}$ and $300 \mathrm{~K}$ respectively. The location of the jet was shown in Figure 3 while the sampling probe was fixed downstream of the jet, as shown in Figure 5. The nano-particles were put into the $\mathrm{CO}_{2}$ jet and the planar laser scattering was used to visualize the flow-field of $\mathrm{CO}_{2}$ jet. According to the work mentioned above, $\mathrm{H}-\mathrm{S}$ tube was used as high frequency actuator and was put into the traditional jet device, which called the excited jet, in order to generate the jet with fluctuation at a certain frequency. This article studied the effects of the excited jet on the mixing in the Mach 2.5 supersonic cross flow.

\section{Results}

\section{A. Instantaneous scattering images}




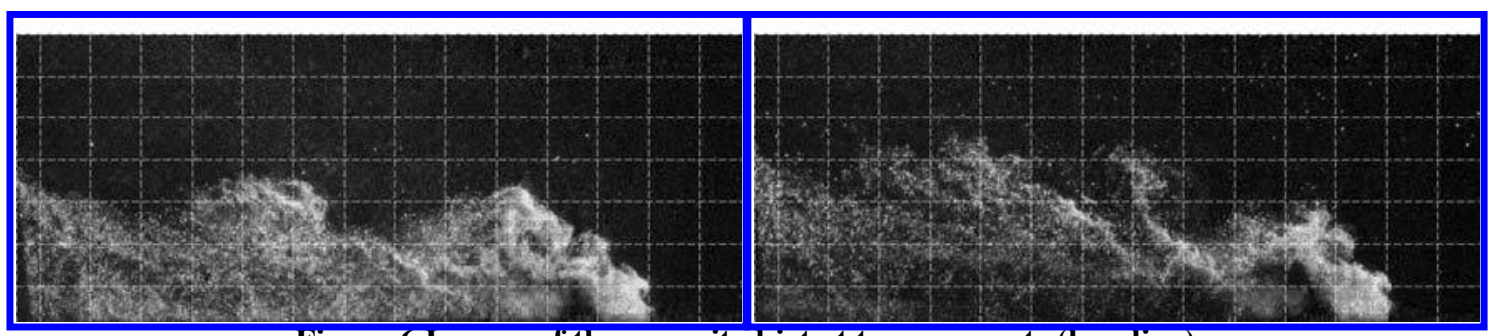

Figure 6 Images of the unexcited jet at two moments (baseline)

The flow structure of unexcited jet at two different moments was shown in Figure 6. The maximum depth of penetration of the $\mathrm{CO}_{2}$ jet was about $3 \mathrm{D}_{\mathrm{e}} \sim 4 \mathrm{D}_{\mathrm{e}}$. There were large-scale structures in the jet shear layer, whose wavelength was between $5 \mathrm{D}_{\mathrm{e}} \sim 6 \mathrm{D}_{\mathrm{e}}$. The depth of penetration would alternate between the big and the small with the large-scale structures existing, and there were also lots of small-scale vortexes in the jet shear layer due to the Kelvin-Helmholtz instability. The $\mathrm{CO}_{2}$ jet would be spread into the supersonic cross flow by those small-scale vortexes which transported by the large-scale structures. Above all, the large-scale structures took big effects on the process and the extent of the mixing between $\mathrm{CO}_{2}$ and air.

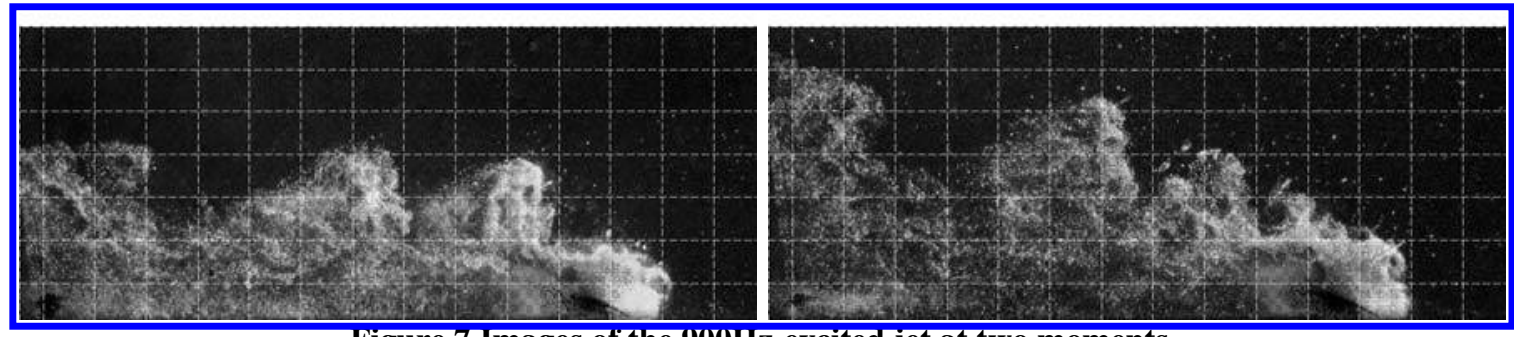

Figure 7 Images of the 900Hz-excited jet at two moments

The images of the jet excited at $900 \mathrm{~Hz}$ were shown in Figure 7. The case of the jet excited at $1.3 \mathrm{kHz}$ was similar to this one. The penetration of the $\mathrm{CO}_{2}$ jet became much deeper than the base one, which increased to 4De 6De. Compared to the base one, the number of the large-scale structures also increased.

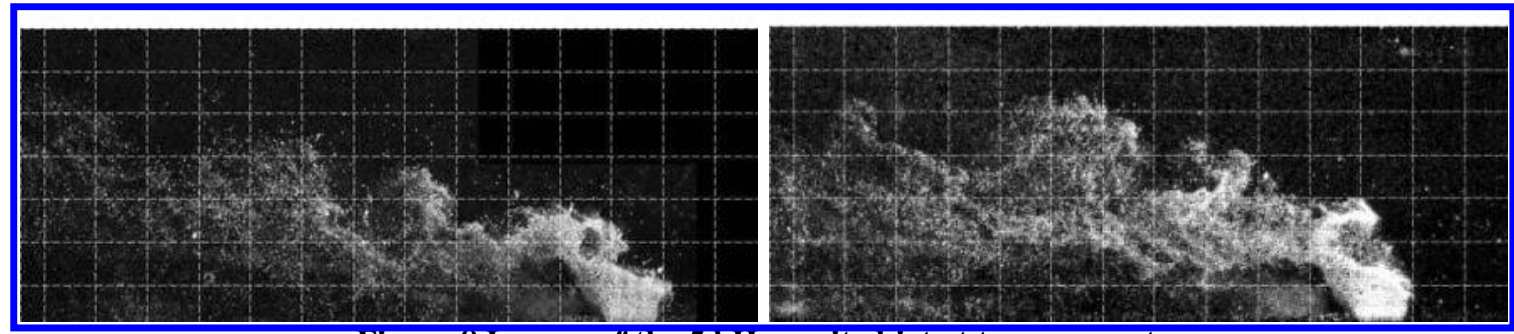

Figure 8 Images of the $5 \mathrm{kHz}-\mathrm{excited}$ jet at two moments

The mixing of the jet excited at $5 \mathrm{kHz}$ was shown in Figure 8. The penetration in this case was deeper than the base one, but little less than one of the $900 \mathrm{~Hz}$-excited case. The number of the large-scale structures was the most of all the case.

According to the results of scattering, the following conclusion could be obtained. First, the mixing was better in the case of the excited jet because of the deeper penetration and the more large-scale structures. Second, the penetration was the deepest in the $900 \mathrm{~Hz}$-excited case while the number of the large-scale structures was the most in the $5 \mathrm{kHz}$-excited case. Third, the excited jet took effects on the jet shear layer.

\section{B. The profile of total pressure loss and the $\mathrm{CO}_{2}$ concentration}

The information of the instantaneous flow was obtained from the scattering images, and the time-averaged information could be obtained from the profile of total pressure loss and $\mathrm{CO}_{2}$ concentration. 

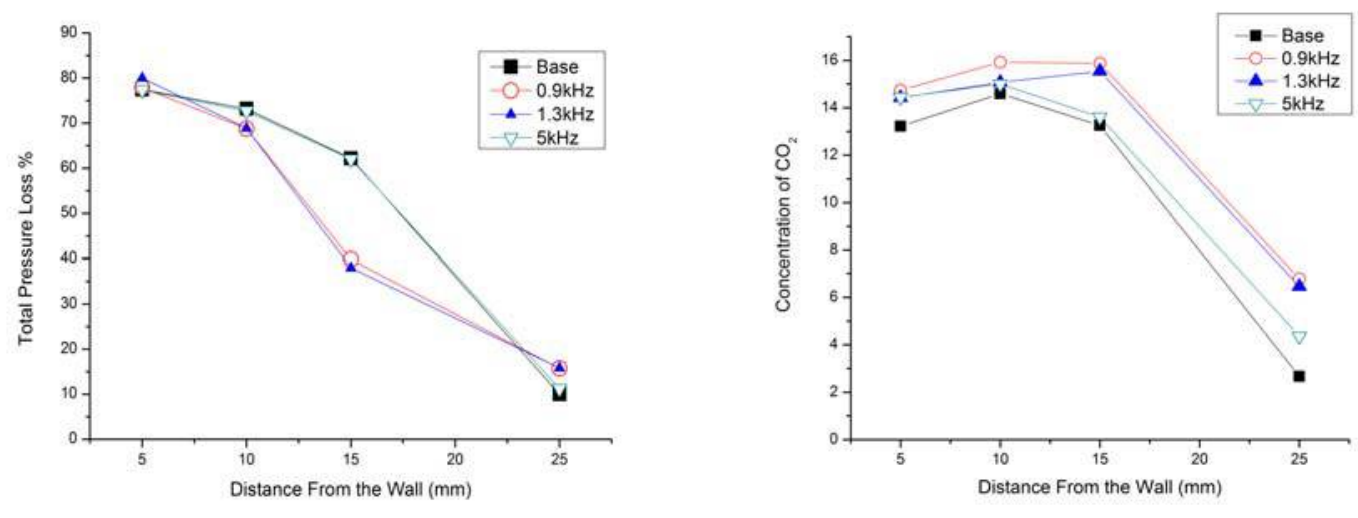

Figure 9 The profile of total pressure loss Figure 10 The profile of $\mathrm{CO}_{2}$ concentration

The probe location was shown in Figure 5. The profile of the total pressure loss and $\mathrm{CO}_{2}$ concentration were shown in Figure 9 and Figure 10 respectively. The $\mathrm{CO}_{2}$ concentration at all four points was bigger in the excited case than in the base. The total pressure loss were close in all the cases at the most points, but the total pressure loss in the $900 \mathrm{~Hz}$-excited and $1.3 \mathrm{kHz}$-excited cases were only half of the base at the point of $15 \mathrm{~mm}$ away from the wall. Therefore, the results shown that the penetration in the excited cases were deeper than base with less total pressure lose.

The maximum of the $\mathrm{CO}_{2}$ concentration in the $900 \mathrm{~Hz}$ - and $1.3 \mathrm{kHz}$-excited cases was shifted from $10 \mathrm{~mm}$ away from the wall to $15 \mathrm{~mm}$ away from the wall. It might result from the flow lifted by the boundary layer due to the excited jet.

\section{Conclusion}

The experimental investigation were carried out for the excited jet into M2.5 flow. According to the scattering images and the profile of the total pressure loss and $\mathrm{CO}_{2}$ concentration, the following conclusion could be:

1) The better mixing with less total pressure loss could be obtained in the excited cases, such as the deeper penetration, the more large-scale structures and the bigger $\mathrm{CO}_{2}$ concentration.

2) The penetration was the deepest in the $900 \mathrm{~Hz}$-excited case while the number of the large-scale structures was the most in the $5 \mathrm{kHz}$-excited case.

3) The excited jet didn't only take effects on the jet shear layer, but also influenced the boundary layer.

\section{Acknowledgments}

The project is funded by the National Natural Science Foundation of China (90305022, 10525212, 10621202).

\section{References}

[1] H. Randolph, L. Chew, and H. Johari, "Pulsed jets in cross flow" J. Propul. Power 10, 7461994

[2] S. Murugappan and E. Gutmark, "Control of penetration and mixing of an excited supersonic jet into a supersonic cross stream", Physics of Fluids 17, 106101, 2005

[3] Virendra Sarohia, Lloyd H.Back, "Experimental Investigation of flow and heating in a resonance tube”, J. Fluid Mech. Vol. 94, pp. 669-672

[4] S. Gu, Lihong Chen, Xinyu Chang, "Numerical Simulation and Experimental Investigation of Excited Oscillation in HS Tube", Journal of Experiments in Fluid Mechnics, 2009 (accepted) 